\title{
Efecto de una intervención basada en el modelo de Educación Deportiva sobre variables psicológicas en nadadores federados
}

\author{
Effect of a Sport Education season on psychological \\ variables in youth swimmers
}

\section{O efeito modelo Educaçáo Desportiva em variáveis psicológicas em nadadores federados}

\author{
Lourdes Merońo ${ }^{1}$, Antonio Calderón ${ }^{1}$ y Peter Hastie ${ }^{2}$ \\ ${ }^{1}$ Facultad de Deporte. Universidad Católica de Murcia (UCAM) y ${ }^{2}$ Department of Kinesiology. Auburn University
}

Resumen: Las aplicaciones prácticas del modelo de Educación Deportiva son del todo conocidas en el contexto escolar y están presentes en numerosas investigaciones. Sin embargo, no se ha reportado información sobre su efecto en el contexto extraescolar o deportivo, ni en deportes con predominio técnico como es la natación. Por ello, se realizó un estudio piloto con diseño cuasi-experimental en nadadores federados $(n=24)$ de diferentes categorías (benjamín, alevín e infantil). El programa de intervención, basado en la Educación Deportiva, se compuso de 16 sesiones de entrenamiento. $\mathrm{Al}$ analizar pre y post las variables objeto de estudio (Percepción de soporte de autonomía; Grado de disfrute y competencia percibida; y Grado de compromiso deportivo) se observaron mejoras estadísticamente significativas. Tal comportamiento fue posteriormente reforzado al diseñar un modelo de ecuaciones estructurales en el que se hipotetizó que la percepción de soporte de autonomía predice positivamente el grado de disfrute y competencia percibida, y con ello, el grado de compromiso deportivo de los jóvenes nadadores. Además, se analizaron las entrevistas realizadas a los nadadores y el diario del entrenador mediante el software NVivo10, para conocer los principales indicadores sobre la percepción de los nadadores (responsabilidad, aprendizaje técnico e implicación) y del entrenador (trabajo en equipo, comunicación y predisposición). Los resultados, que corroboran los reportados en el contexto escolar, mostraron un comportamiento favorable de las variables psicológicas objeto de estudio tras la intervención basada en la Educación Deportiva con nadadores, lo que da indicios sobre su idoneidad también en el contexto federativo.

Palabras clave: Educación Deportiva, club deportivo, natación, variables psicológicas

Abstract: The practical applications of Sport Education in the school context are fully known in the research of the last twenty years. However, it is not reported its effects on extracurricular or sporting context but also in sports with technical dominance as swimming. A pilot study with quasiexperimental design was performed in youth swimmers $(n=24)$ of different categories (junior and youth). The intervention program based on the Sport Education, consisted of 16 training sessions. By analyzing the variables under study (Perceived Autonomy Support, Enjoyment and Perceived Competence, and Sport Commitment) statistically significant improvements were observed. Such behavior was further reinforced when designing a structural equation model in which it was hypothesized that perceived autonomy support positively predicts the enjoyment and perceived competence, and the sport commitment of the swimmers. Interviews and diary coaches' logs were analyzed by NVivo10 software to know the perception of swimmers (responsibility, technical learning and involvement) and coaches (teamwork, communication and willingness). The main results (consistent with the school context ones) showed an optimal behavior of the psychological variables of this study after the Sport Education intervention. This reinforces the benefits of Sport Education also in the youth sport context.

Keywords: Sport Education, youth sport, swimming, psychological variables.

Resumo: As aplicaçôes práticas da Educação Desportiva no contexto escolar são totalmente conhecidos e estão presentes em inúmeras investigaçôes. No entanto, não se sabe qual o efeito do contexto esportivo em desportes ou domínio técnico, é a natação. Portanto, um estudo piloto com desenho quase-experimental foi realizada em nadadores federados $(n=24)$ de diferentes categorias (bebê, juvenil e infantil). O programa de intervenção com base na Educação Desportiva, consistiu em 16 sessões de treinamento. Ao analisar as variáveis em estudo (apoio autonomia percebido; Grau de satisfação e percepção de competência, e grau de compromisso esporte) estatisticamente foram observadas melhorias significativas. Tal comportamento foi reforçado quando a concepção de um modelo de equações estruturais em que foi levantada a hipótese de que o apoio autonomia percebida prediz positivamente o grau de satisfação e percepção de competência e, portanto, o grau de comprometimento do esporte de jovens nadadores. Além disso, foram analisadas entrevistas com nadadores e treinador diária por software NVivo10. Assim, os principais indicadores sobre a percepçáo dos nadadores (responsabilidade, de aprendizagem técnica e envolvimento) e treinador (trabalho em equipe, comunicação e vontade) se encontraram. Os resultados mostraram o bom desempenho das variáveis psicológicas do estudo, após a aplicação da Educação Desportiva, no âmbito federal (natação); sendo consistente com os resultados anteriormente encontrados no contexto escolar.

Palavras-chave: esporte educação, saúde clube, natação, variáveis psicológicas. 


\section{Introducción}

La investigación sobre la eficacia del proceso de enseñanza y aprendizaje en educación física, sigue siendo un tópico de interés en las revistas especializadas desde Locke (1977). Los trabajos de Ennis (2014), Lindsay (2014), Metzler (2014), Roberts (2014), Solmon y Garn (2014), son un claro ejemplo de ello. Sin embargo no ha estado tan presente en la literatura que la analiza en un contexto extraescolar o deportivo (Gilbert y Trudel, 2004). Los estudios que predominan en este contexto, se centran en conocer las sinergias entre las principales variables de entrenamiento y las variables psicológicas que optimizan el rendimiento deportivo (Bompa, 2003; Platonov y Bulatova, 2007). En el contexto escolar no obstante, está definida por estudios que se centran en conocer el efecto de determinadas variables de proceso, sobre la experiencia de aprendizaje de los alumnos (Silverman y Manson, 2003). Una de las variables que se analiza, es el modelo de enseñanza. Entre los más analizados están el Modelo Comprensivo (Bunker y Thorpe, 1982), el Modelo Cooperativo (Dyson y Casey, 2012) y en mayor medida la Educación Deportiva (Siedentop, 1994). Una de las tendencias de investigación que se está consolidando en los estudios actuales sobre la Educación Deportiva, analiza la relación entre algunas variables psicológicas y su influencia sobre el aprendizaje del alumno en educación física (Hastie, Martínez de Ojeda y Calderón, 2011). Así, en los estudios de Perlman y GocKarp (2010), y Wallhead y Ntoumanis (2004) se reportó un incremento en la motivación intrínseca de los alumnos, debido al clima-tarea que se fomenta durante la práctica. Incremento basado fundamentalmente en el comportamiento autónomo que genera y en la mejora de la percepción de competencia (Wallhead, Hagger y Smith, 2010). Estos resultados han sido corroborados por Perlman (2010), Sinelnikov y Hastie (2010), Sinelnikov, Hastie, y Prusak (2007), Spittle y Byrne (2009). Además, los alumnos que experimentan una (o varias) unidades didácticas basadas en la Educación Deportiva, manifiestan un elevado grado de autonomía (Calderón, Martínez de Ojeda, y Martínez, 2013); de disfrute (MacPhail, Gorely, Kirk, y Kinchin, 2013) y de competencia percibida (Calderón, Martínez de Ojeda, y Hastie, 2013), lo que aumenta las posibilidades de adherencia y/o compromiso de práctica en un contexto extraescolar o deportivo (Wallhead, et al., 2010), si bien se precisan más investigaciones de tipo longitudinal en esta línea (Hastie, et al., 2011). Las aplicaciones prácticas, y resultados del desarrollo de la Educación Deportiva en la clase de educación física son múltiples y han sido abordadas en dos revisiones sistemáticas (Hastie, et al., 2011; y Wallhead y O’Sullivan, 2005), y en numerosos trabajos de investigación desde 1994. Los contenidos que se han abordado fundamentalmente son deportes en los que existe un mayor predominio táctico. Sin embargo, las aplicaciones prácticas y resultados de la Educación Deportiva son del todo desconocidas en un contexto extraescolar o deportivo, y menos frecuentes en deportes con un mayor predominio técnico. Es por ello por lo que en el presente trabajo piloto se analiza el efecto de un programa de intervención basado en la Educación Deportiva, sobre la percepción de soporte de autonomía, el grado de disfrute y competencia percibida, y el grado de compromiso deportivo en un grupo de deportistas pertenecientes a un club deportivo de natación.

\section{Método}

\section{Participantes}

El estudio piloto se desarrolló en un club deportivo de natación con 24 nadadores federados, 11 chicos y 13 chicas de edades comprendidas entre los ocho y los 16 años $(M=11.54$; $D T=2.94)$, pertenecientes a las categorías benjamín, alevín e infantil. Se contó con el consentimiento informado de la Junta directiva del club, y de los padres y las madres de los nadadores implicados. La intervención fue dirigida por tres entrenadores: una entrenadora con más de 30 años de experiencia; un entrenador de 15 años de experiencia; y una entrenadora con tres años de experiencia en el alto rendimiento deportivo en natación. Los tres entrenadores con la licenciatura en ciencias de la actividad física y del deporte; y la titulación superior de natación (entrenador nacional).

\section{Diseño y procedimiento}

Se realizó un diseño cuasi experimental intragrupo (Thomas, Nelson y Silverman, 2011). El programa de intervención se diseñó teniendo en cuenta las premisas de la Educación Deportiva (Tabla 1). 
Tabla 1. Contenidos del programa de intervención basado en las premisas dela Educación Deportiva. Tipos de entrenamiento según la nomenclatura de Maglischo (2009).

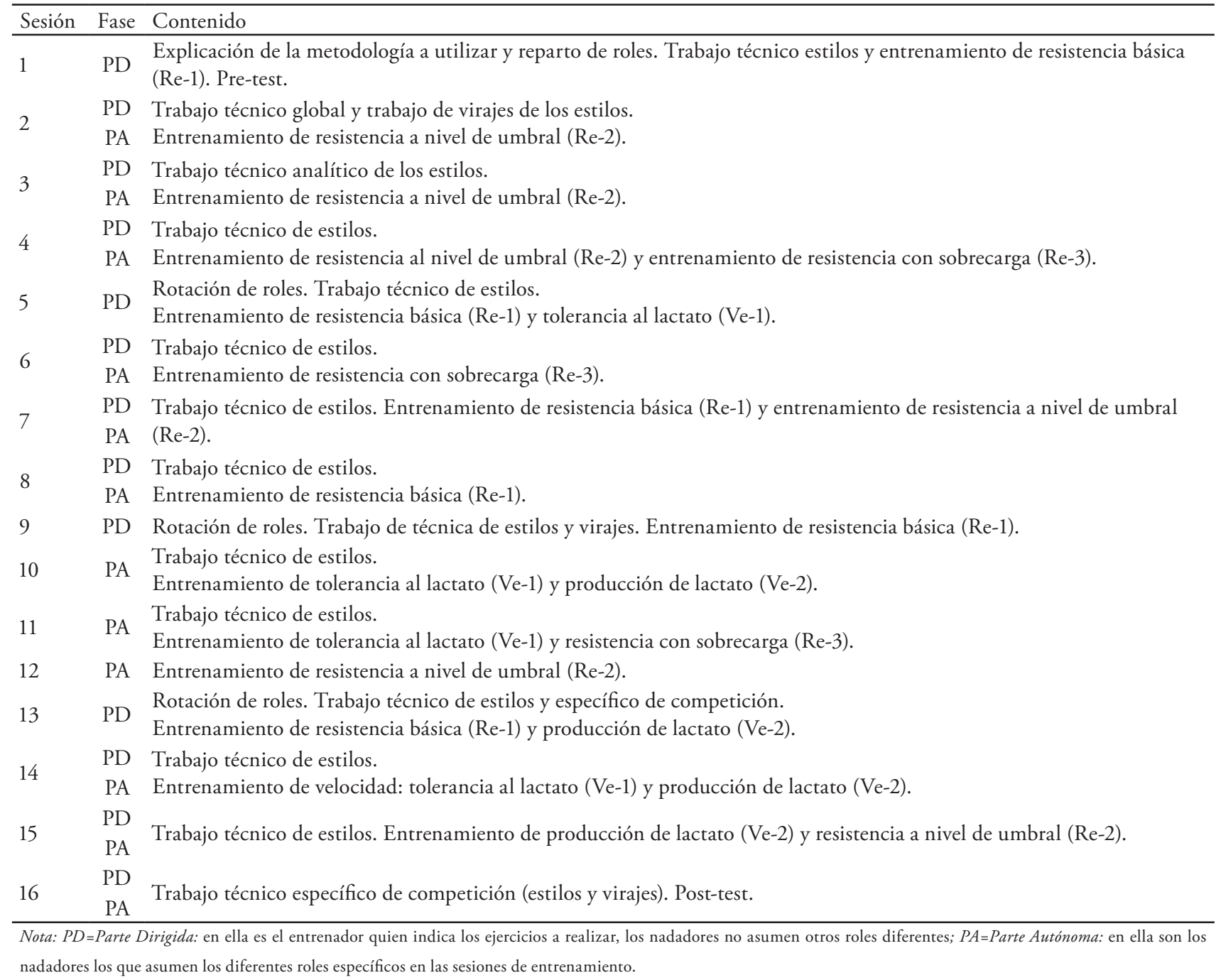

Se prolongó durante cuatro semanas por un total de dieciséis sesiones de dos horas y media cada una, siempre desarrolladas bajo la supervisión del entrenador principal. Los contenidos abordados se centraron en el trabajo técnico de los estilos de natación y la condición física de los jóvenes deportistas. Se siguieron las pautas recomendadas por Sinelnikov (2009) para asegurar la fiabilidad de la intervención. De modo que: el entrenador principal recibió la formación teórica y práctica específica sobre el modelo Educación Deportiva, se emplea- ron materiales didácticos propios del modelo (hojas de registro y evaluación) y se comprobó que las principales características del mismo se cumplían durante el desarrollo de las sesiones de entrenamiento. Tal como recomendaron Hastie, et al. (2011) se establecieron roles específicos para el contexto de la natación federada (Tabla 2): entrenador, cronometrador, responsable del ranking, responsable del material, observador técnico y periodista. Todos los nadadores asumían dos roles: el rol de nadador y un rol específico. 
Tabla 2. Descripción de las funciones de cada rol específico.

\begin{tabular}{ll}
\hline Entrenador & $\begin{array}{l}\text { Indica a sus compañeros los ejercicios que hay que realizar y se encarga de resolver las dudas que puedan surgir } \\
\text { durante la sesión de entrenamiento. }\end{array}$ \\
$\begin{array}{l}\text { Cronometrador } \\
\text { Responsable del ranking }\end{array}$ & $\begin{array}{l}\text { Registra los tiempos de cada nadador en la pizarra de entrenamiento y contabiliza el sumatorio de puntos pro- } \\
\text { cedentes de las hojas de observación en el póster también expuesto en la pizarra. }\end{array}$ \\
Responsable del material & $\begin{array}{l}\text { Expone cada día en la pizarra un póster informativo con las funciones de cada rol, un cronograma con las } \\
\text { funciones correspondientes a cada nadador; y organiza el material necesario para la sesión (por ejemplo: tablas } \\
\text { y pull-buoys). }\end{array}$ \\
Observador técnico & $\begin{array}{l}\text { Al finalizar la sesión de entrenamiento valora mediante hojas de observación la ejecución técnica de los nadado- } \\
\text { res (mariposa, espalda, braza y crol). } \\
\text { Realiza fotografías a sus compañeros a lo largo de la sesión de entrenamiento. }\end{array}$ \\
\hline
\end{tabular}

La intervención comenzó con una explicación por parte del entrenador de la metodología de trabajo a desarrollar, de los roles a asumir y la asignación semanal de los mismos. El primer día de cada semana de entrenamiento se planteó una sesión dirigida por el entrenador principal. En ella, además de los ejercicios diarios, se realizó una rotación de roles entre los nadadores y se producía la toma de contacto con el rol semanal correspondiente que el entrenador asignaba. La primera y segunda semana, las sesiones se caracterizaron por disponer de una primera parte de la sesión dirigida y una segunda parte autónoma, en la cual cada nadador asumía el rol específico semanal asignado. La tercera semana, se caracterizó por su estructura totalmente autónoma (nadadores asumen los roles individuales y específicos). La cuarta, y última semana del programa de intervención, se volvió a la estructura de la primera semana (primera parte dirigida y una segunda autónoma).

\section{Recogida de datos e instrumentos}

Tal como recomienda Maxwell (2013), para lograr una mayor frabilidad de los resultados, se utilizaron instrumentos de recogida de datos cuantitativos (cuestionarios) y cualitativos (entrevistas de los nadadores y diario del entrenador). Las variables dependientes analizadas (percepción de soporte de autonomía, disfrute y competencia percibida, y grado de compromiso deportivo) fueron valoradas al inicio y al final del programa de intervención (pre-test y post-test). Los cuestionarios utilizados fueron:

1.- Cuestionario de Percepción de Soporte de la Autonomía en el Proceso de Entrenamiento ASCQ, en jóvenes deportistas españoles. Se utilizó la versión validada por Conde, et al. (2010). Se compone de dos dimensiones: interés en la opinión del deportista (cinco ítems), y valoración del comportamiento autónomo (cuatro ítems). Un total de nueve ítems a completaren escala tipo Likert de uno a cinco.

2.- Escala de disfrute y competencia percibida en Natación. Se utilizó la versión adaptada a la natación del cuestionario de Arias, Alonso, y Yuste (2013). Se compone de dos dimensiones: grado de disfrute (tres ítems) y competencia percibida (cuatro ítems). Un total de siete ítems a completar en escala tipo Likert de uno a cinco.

3.- Escala de grado de compromiso deportivo. Se utilizó el cuestionario validado por Belando, Ferriz-Morell, y Moreno-Murcia (2012). Este cuestionario originalmente se compone de once ítems en escala tipo Likert de uno a cinco; y dos dimensiones: compromiso de práctica actual (siete ítems) y el compromiso de práctica futura (cuatro ítems). Se compuso de diez ítems totales; y las dos dimensiones: compromiso de práctica actual (siete ítems), y compromiso de práctica futura (tres ítems).

4.- Entrevistas. El guión de la entrevista se adaptó del utilizado por Kinchin, MacPhail, y Ni Chroinin (2009) y se siguieron las indicaciones de Ennis y Chen (2012) (Tabla 3). Fueron realizadas al finalizar el programa de intervención. Las preguntas trataron de provocar en los nadadores la expresión de su percepción a propósito de cada una de las variables analizadas en los cuestionarios.

Tabla 3. Guión básico de la entrevista realizada a los nadadores para finalizar el programa de intervención.

1.- ¿`Te parece bien entrenar asumiendo roles dentro del equipo? (Autonomía)

2.- ¿Me podrías hablar de tu diversión y de tu percepción de competencia con la metodología anterior y con esta? (Disfrute y percepción de competencia).

3.- ¿Me podrías hablar de tu motivación en los entrenamientos con la metodología anterior y esta?

(Compromiso deportivo)

4.- ¿Qué es lo que más te ha gustado de esta metodología? ¿y lo que menos?(General)

5.- Diario del entrenador. Siguiendo las premisas de Jurado (2011) se utilizó un diario de estilo abierto (Barbier, 1997), en el que el entrenador cada día de forma libre, debía incluir todos los aspectos, observaciones y comportamientos que consideraba de vital importancia a lo largo del programa de intervención con el modelo Educación Deportiva. 


\section{Análisis de datos}

En primer lugar se calcularon los estadísticos descriptivos (medias y desviaciones típicas) de las diferentes variables dependientes del estudio (autonomía, grado de disfrute y competencia percibida, y compromiso deportivo) y también se realizaron correlaciones bivariadas mediante IBM SPSS v20. A continuación se realizó un modelo de ecuaciones estructurales para analizar las relaciones hipotetizadas entre las variables de estudio. Se precisa destacar que de forma previa a este análisis se comprobó la validez de los instrumentos utilizados en el estudio a través de un modelo de medición, tal como formalizaron Moreno-Murcia, Cervelló-Gimeno, Marcos-Pardo y Martín-Dantas (2010). Tanto el modelo de medición como los modelos de ecuaciones estructurales se valoraron mediante AMOS v18. Para ello, se siguió el procedimiento determinado por Cervelló, Santos-Rosa, Jiménez, Nerea, y García (2010); donde se ejecutaban modelos de ecuaciones estructurales sobre variables psicológicas también presentes en un contexto deportivo. Además, para complementar tales resultados se realizó análisis del contenido tanto de todas las entrevistas como del diario del entrenador, se transcribieron y se analizaron con el programa QSR NVivo 10. Siguiendo las recomendaciones de Bardin (2002), se realizó un sistema de codificación tras una primera lectura superficial (exploratoria) para crear categorías e indicadores a través de un razonamiento inductivo. El objetivo del análisis de contenido, tanto de las entrevistas como del diario, fue conocer la percepción de los nadadores sobre su experiencia tras el programa de intervención.

\section{Resultados}

\section{Análisis descriptivos, correlaciones bivariadas y análisis de} fiabilidad

En este apartado se presentan los estadísticos descriptivos y las correlaciones obtenidas entre la autonomía, el grado de disfrute y competencia percibida, y el grado de compromiso deportivo (Tabla 4). Tal como se puede apreciar al finalizar la intervención el valor medio de la percepción de soporte de autonomía de los nadadores es menor, que el grado de disfrute y competencia percibida y que el grado de compromiso deportivo. Además se comprueba que existe correlación altamente positiva entre la percepción del soporte de autonomía y el grado de disfrute y competencia percibida $(p=.004)$; y este último a su vez presentaba correlación significativa con el grado de compromiso deportivo $(p=.000)$.

Tabla 4. Estadísticos descriptivos y correlaciones de las variables de estudio ( $n=24)$.

\begin{tabular}{lcccc}
\hline Variables & $\mathrm{M}$ & $\mathrm{DT}$ & $(2)$ & $(3)$ \\
\hline (1) Autonomía & 3.43 & 0.50 & $0.56^{* *}$ & $0.47^{*}$ \\
(2) Grado de disfrute y competencia & 4.36 & 0.56 & - & $0.77^{* *}$ \\
percibida & 4.30 & 0.54 & - & - \\
(3) Compromiso deportivo & & &
\end{tabular}

Escala 1(mínimo) 5(máximo)

*Nivel de significación para $\mathrm{p}<0.05$. ${ }^{* *}$ Nivel de significación para $\mathrm{p}<0.01$.

Las tres variables psicológicas de estudio presentaron mejoras estadísticamente significativas tras la aplicación del programa de intervención con la Educación Deportiva (Tabla 5). Además, los coeficientes de consistencia interna de las tres escalas conseguían valores aceptables (Carretero-Dios y Pérez, 2007). Tras realizar el análisis factorial, se optó por eliminar un ítem perteneciente a la dimensión de compromiso de práctica futura, para optimizar la fiabilidad ya que presentaba un valor de 0.47 de Alpha de Cronbach. Los resultados sobre el indicador "interés en la opinión del deportista” presentaron valores próximos a la media e inferiores al resto de los indicadores de estudio. Fundamentalmente porque los ítems que lo definen hacen referencia a la participación de los nadadores en el diseño de las tareas y los ejercicios que se llevaron a cabo durante la sesión de entrenamiento, aspecto que no se contempló en el presente estudio piloto por la inexperiencia de los nadadores en esta línea.

Tabla 5. Estadísticos descripticos y coeficientes de consistencia interna de los cuestionarios de estudio $(n=24)$.

\begin{tabular}{lcccc}
\hline Variables & M Pre(DT) & M Post(DT) & $p$ & Coeficiente Alpha de Cronbach \\
\hline (1)Autonomía & $2.54(0.72)$ & $4.42(0.65)$ & $.000^{*}$ & \\
Interés en la opinión del deportista & $2.00(0.59)$ & $2.72(0.67)$ & .007 & 0.71 \\
Valoración del comportamiento autónomo & $3.46(0.83)$ & $4.25(0.53)$ & .042 & 0.75 \\
(2)Grado de disfrute y competencia percibida & $3.83(0.81)$ & $4.38(0.71)$ & $.000^{*}$ & \\
Grado de disfrute & $4.56(0.93)$ & $4.79(0.50)$ & .031 & 0.79 \\
Competencia percibida & $3.42(0.83)$ & $4.00(0.59)$ & $.000^{*}$ & 0.81 \\
(3)Compromiso deportivo & $3.92(0.65)$ & $4.29(0.75)$ & $.001^{*}$ & \\
Compromiso de práctica actual & $3.92(0.65)$ & $4.46(0.77)$ & $.000^{*}$ & 0.80 \\
Compromiso de práctica futura & $3.87(0.68)$ & $4.00(0.59)$ & $.000^{*}$ & 0.68 \\
\hline
\end{tabular}

Escala 1(mínimo) 5(máximo). ${ }^{*}$ Nivel de significación para $\mathrm{p}<0.05$. 
Modelo de medición

Se comprobó la relación existente entre las variables de estudio mediante el método de los dos pasos (modelo de medición y modelo de ecuaciones estructurales) propuesto por Anderson y Gerbing (1988). En primer lugar se realizó el análisis factorial confirmatorio (CFA) y se testó la validez del constructo (Perugini y Conner, 2000). Tal como propusieron Moreno-Murcia, et al. (2010), se parcelaron los ítems de cada factor o variable de estudio en dos grupos homogéneos. Posteriormente, se procedió a la elaboración del modelo siguiendo los pasos de Ruiz, Pardo, y San Martín (2010): se formuló la teoría que lo sustenta y se especificaron las relaciones que se esperaba encontrar entre variables.

Figura 1. Modelo de medición (CFA). Los círculos representan los constructos latentes y los rectángulos las variables medidas (grupos aleatorios de ítems).

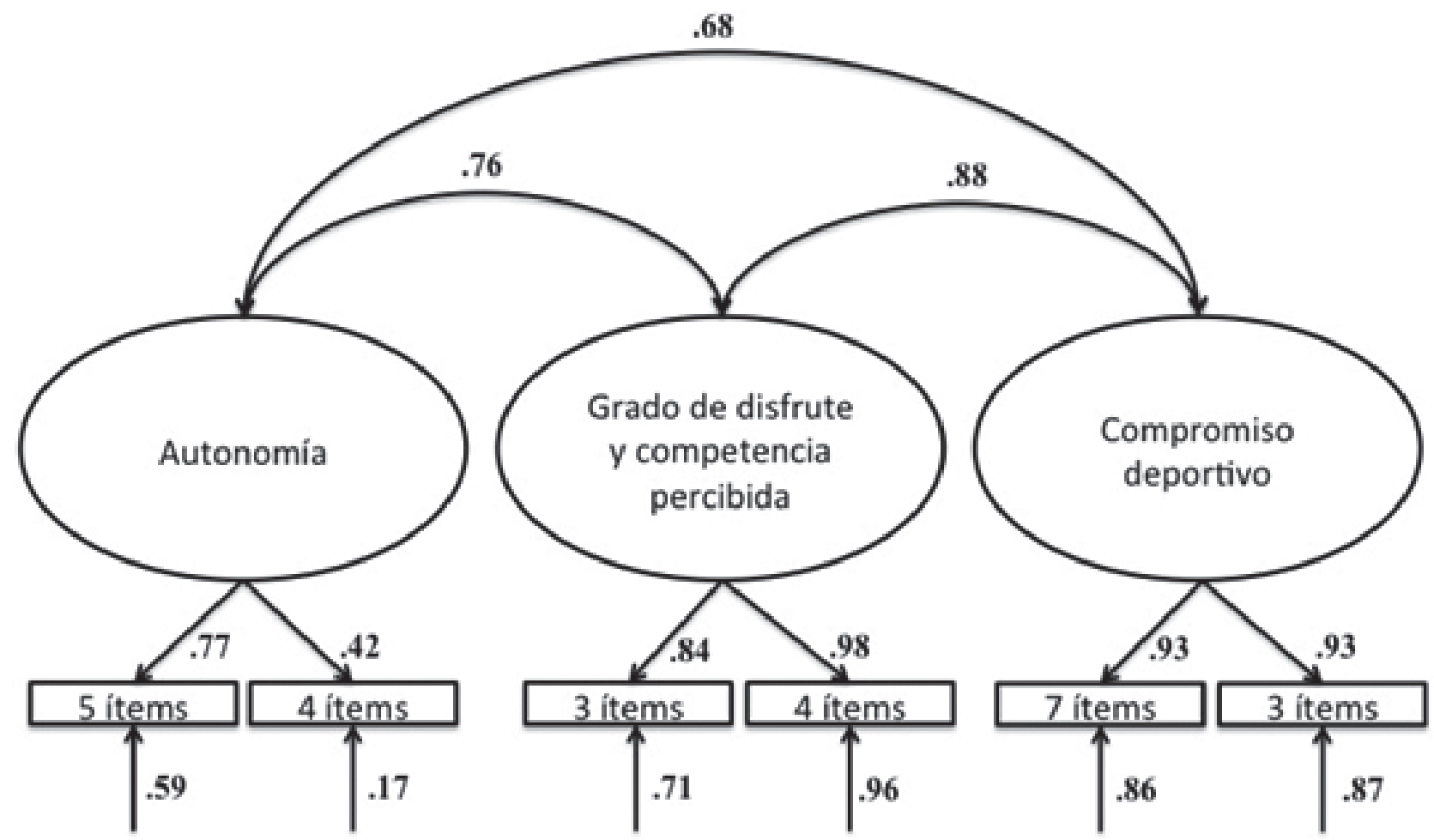

Todos los parámetros fueron estandarizados y estadísticamente significativos. Las varianzas se muestran sobre las flechas pequeńas. Una vez que se estimó el modelo, se calcularon todos los parámetros estadísticos de bondad de ajuste, tal como indicaban Schreiber, Nora, Stage, Barlow, y King (2006). Los índices fit ó índices de bondad de ajuste, que se consideraron para valorar la bondad del modelo de ecuaciones estructurales fueron: $X^{2}, X^{2} / g . l$, RMSEA (Root Mean Square Error of Aproximation), SRMR (Standardized Root Mean Square Residual) y los índices incrementales (CFI, NFI y TLI). Estos índices de bondad de ajuste se consideran aceptables cuando el valor de $X^{2} / g . l$. sea inferior a 5 (Bentler, 1989), los índices incrementales (CFI, NFI y TLI) superiores a .95, el índice de error RMSEA inferior a .08, el índice de error SRMR próximo a cero, y el valor de $p$ mayor de .05 (Ruiz, et al., 2010). Los índices fit o índices de bondad de ajuste obtenidos en el modelo de medición $\left[X^{2}(21, N=24)=101.12, p=7.26, X^{2}\right.$ d.f.=3.63, CFI=1.00, IFI=1.02, TLI=1.06, SRMR=.08, RM$\mathrm{SEA}=.00)$ ], se ajustaron a los parámetros establecidos, por lo que el modelo propuesto se consideró válido. Los resultados del modelo de medición revelaron la interrelación existente de la percepción de soporte de autonomía con el grado de disfrute y competencia percibida $(\beta=.76)$, y este último a su vez con el grado de compromiso deportivo $(\beta=.88)$. También se halló la relación existente entre la autonomía y el grado de compromiso deportivo $(\beta=.68)$.

Modelos de ecuaciones estructurales

Se testó simultáneamente el modelo estructural y de medición, para así comprobar las interacciones conceptuales exis- 
tentes entre los factores latentes y las variables. El contenido del modelo propuesto trató de valorar las interacciones hipotetizadas que existían entre la valoración de la autonomía, el grado de disfrute y competencia percibida, y con ello el grado de compromiso deportivo. Los resultados de este modelo reflejaron índices de ajuste dentro de un rango aceptable (Ruiz, et al., 2010): $\mathrm{X}^{2}(21, N=24)=101.12 ; p=.820 ; \mathrm{X}^{2} /$ d.f. $=3.64$; $\mathrm{CFI}=1.00 ; \mathrm{IFI}=1.03 ; \mathrm{TLI}=1.08 ; \mathrm{RMSEA}=.00 ; \mathrm{SRMR}=.08$. (Figura 2).

Los resultados del análisis del modelo de ecuaciones estructurales revelaron una relación positiva entre la valoración del comportamiento autónomo del nadador, el grado de disfrute y competencia percibida, y el compromiso deportivo. La valoración del comportamiento autónomo se encontró positivamente relacionada con el grado de disfrute y competencia percibida $(\beta=.76)$, y a su vez el grado de disfrute y competencia percibida se relacionaba de forma positiva con el grado de compromiso deportivo $(\beta=.88)$.

\section{Percepción de los nadadores y del entrenador}

Tras el análisis del contenido tanto de las entrevistas realizadas a los nadadores como el diario del entrenador, se determinaron los indicadores percibidos sobre las variables psicológicas objeto de estudio (Tabla 6).
Figura 2. Modelo de ecuaciones estructurales mostrando las relaciones entre la valoración de las variables psicológicas de estudio. Los parámetros están estandarizados y son estadísticamente significativos. Las varianzas se muestran sobre las flechas pequeńas.

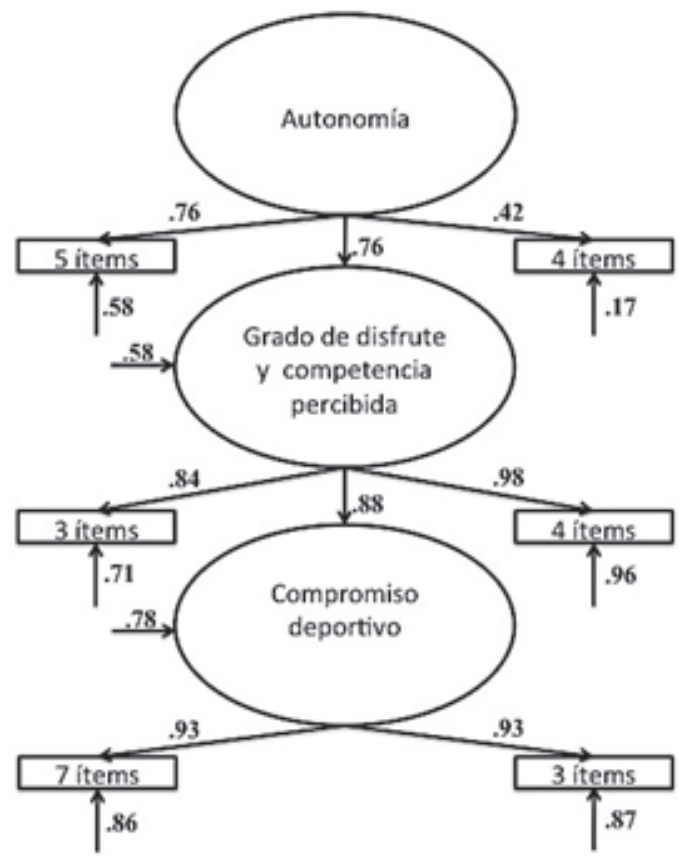

Tabla 6. Percepción de los nadadores y del entrenador sobre la intervención basada en el modelo Educación Deportiva.

\begin{tabular}{|c|c|c|c|c|}
\hline \multirow{2}{*}{ Variables Psicológicas } & \multicolumn{2}{|l|}{ Entrevistas (Nadador) } & \multicolumn{2}{|l|}{ Diario (Entrenador) } \\
\hline & Indicadores & Frecuencia* & Indicadores & Frecuencia \\
\hline \multirow{5}{*}{ Percepción de Autonomía } & Responsabilidad & $54.23 \%$ & \multirow{5}{*}{$\begin{array}{l}\text { Trabajo en equipo } \\
\text { Responsabilidad } \\
\text { Libertad }\end{array}$} & $40.42 \%$ \\
\hline & Toma de decisión & $16.94 \%$ & & $38.29 \%$ \\
\hline & Comunicación & $13.55 \%$ & & $21.29 \%$ \\
\hline & Autoridad & $10.16 \%$ & & \\
\hline & Libertad & $5.12 \%$ & & \\
\hline \multirow{3}{*}{ Grado de Disfrute } & Satisfacción & $42.85 \%$ & Sentimientos & $44.44 \%$ \\
\hline & Mejora de la autoestima & $38.77 \%$ & Éxito & $30.55 \%$ \\
\hline & Aspecto lúdico & $18.38 \%$ & Diversión & $25.01 \%$ \\
\hline \multirow{4}{*}{ Competencia Percibida } & Aprendizaje técnico & $54.54 \%$ & Conocimiento técnico & $29.23 \%$ \\
\hline & Concienciación & $22.72 \%$ & Superación & $26.15 \%$ \\
\hline & Valoración & $13.63 \%$ & Iniciativa personal & $23.07 \%$ \\
\hline & Competitividad & $9.11 \%$ & Autoestima & $21.55 \%$ \\
\hline \multirow{6}{*}{ Compromiso Deportivo } & Implicación & $35.16 \%$ & Predisposición & $25.39 \%$ \\
\hline & Superación & $25.27 \%$ & \multirow{5}{*}{$\begin{array}{l}\text { Comunicación } \\
\text { Identidad } \\
\text { Respeto } \\
\text { Dedicación }\end{array}$} & $22.22 \%$ \\
\hline & Reflexión & $16.48 \%$ & & $19.04 \%$ \\
\hline & Concentración & $12.08 \%$ & & $19.04 \%$ \\
\hline & Respeto & $7.69 \%$ & & $14.31 \%$ \\
\hline & Progresión & $3.32 \%$ & & \\
\hline
\end{tabular}


Los nadadores destacaron los siguientes indicadores sobre la percepción la autonomía a lo largo de la intervención con la Educación Deportiva: responsabilidad (54.23\%) "Quiero hacer bien mi rol mientras entreno"; toma de decisión (16.94\%) "Continuamente tenemos que ser capaces de dar soluciones"; comunicación (13.55\%) "Hablamos entre nosotros para saber qué debemos hacer a lo largo del entrenamiento"; autoridad (10.16\%) "Me tienen que hacer caso para poder entrenar"; y libertad (5.12\%) "con los roles ya sabemos lo que tenemos que hacer durante el entrenamiento". Al analizar el diario del entrenador, sobre la percepción de autonomía de los nadadores, se acentuaron los siguientes indicadores: trabajo en equipo (40.42\%) "Es increíble el cambio porque ahora todos trabajan juntos y sólo les importa hacerlo bien para ser los mejores"; responsabilidad (38.29\%) "Con la Educación Deportiva asumen sus funciones y les gusta hacer bien su tarea"; y libertad (21.29\%) "Ahora con esta nueva metodología tanto ellos como yo sabemos las funciones de cada uno y debemos actuar".

$\mathrm{Al}$ analizar el grado de disfrute, los nadadores incidían en los indicadores: satisfacción (42.85\%) "Tras cada entrenamiento me voy feliz y quiero contarle a mis padres lo que he hecho"; mejora de la autoestima (38.77\%) "Nos sentimos importantes nadando porque aprendemos y sacamos lo mejor de nosotros"; y el aspecto lúdico (18.38\%) "Ahora parece que entrenamos jugando". La percepción del entrenador plasmada en su diario sobre esta variable fue la siguiente: sentimientos (44.44\%) "Hoy me decian que querian seguir entrenando así porque todos se sienten piezas importantes del equipo"; éxito (30.55\%) "Ha merecido la pena porque ellos han mejorado y han disfrutado; yo también he aprendido con ellos"; y diversión (25.01\%) "Hoy tras cada serie aplaudian al nadador-cronometrador porque sabía cómo motivarlos".

La percepción de competencia percibida también fue manifestada por los nadadores en los siguientes indicadores: aprendizaje técnico (54.54\%) "Las hojas de observación me han ayudado a mejorar mi estilo"; concienciación (22.72\%) "Entro en la piscina pensando qué tengo que hacer"; valoración (13.63\%) "Todos los dias al finalizar nadamos nuestro mejor estilo mientras nos observan para ponernos nota"; y competitividad (9.11\%) "Al terminar siempre queremos saber quien lo hace mejor". El entrenador enfatizó: el conocimiento técnico (29.23\%) "Alobservar a los demás y autoevaluarse, piensan cada gesto técnico mientras aprenden"; la superación (26.15\%) "Es increíble ver como los nadadores mejoran y entrenan con mayor motivación"; la iniciativa personal (23.07\%) "Al terminar la última serie me ha conmovido ver cómo se han organizado"; y la autoestima (21.55\%) "Estoy orgullosa de ver lo que han aprendido mis nadadores".

También se analizó la percepción de los nadadores sobre el grado de compromiso deportivo: implicación (35.16\%) "Me gusta ser entrenador y que me hagan caso"; superación (25.27\%) "Queremos hacer nuestro rol cada día mejor"; reflexión (16.48\%)
"Ahora con las observaciones pienso en mi estilo para saber hacerlo bien"; concentración (12.08\%) "El trabajo técnico lo hago muy despacio y pienso en cada movimiento"; respeto (7.69\%) "Sorprendentemente nos mandamos entre nosotros y nos tomamos en serio"; y progresión (3.32\%) "Tras las observaciones me están diciendo que he mejorado mi estilo de competición”. El entrenador interpretó el compromiso deportivo de los nadadores en los siguientes indicadores: predisposición (25.39\%) "Todos pretenden hacerlo bien e integrarse en el equipo"; comunicación (22.22\%) "Los nadadores comentan cada día todo lo que hacen"; identidad (19.04\%) "Me están sorprendiendo porque se lo toman en serio y se meten verdaderamente en el papel"; respeto (19.04\%) "Curiosamente ahora se respetan más, porque saben que todos son importantes para aprender"; y dedicación (14.31\%) "Están contentos y se implican en cada entrenamiento". En resumen, los resultados del análisis del contenido tanto de las entrevistas como del diario del entrenador, mostraron efectos positivos sobre el comportamiento autónomo de los nadadores, generado por las características de la intervención. Que condicionó el grado de disfrute, la percepción de competencia, y su vez, la implicación y el grado de compromiso deportivo. Por lo que se refuerza el modelo hipotetizado.

\section{Discusión}

El objetivo del presente trabajo fue analizar el efecto de un programa de intervención basado en la Educación Deportiva, sobre la percepción de autonomía; el grado de disfrute y competencia percibida, y el grado de compromiso; en un grupo de nadadores pertenecientes a un club deportivo. Tal como se ha podido comprobar tras el análisis de los resultados, la percepción de autonomía de los jóvenes deportistas se incrementó a lo largo de la intervención. Este resultado ya ha sido encontrado en estudios realizados en el contexto escolar (Hastie, et al., 2011; Hastie, Sinelnikov y Guarino, 2009; Wallhead, et al., 2010). En el contexto federado parece ser que la tendencia es similar, a pesar de que no se encontraron valores elevados en la percepción de interés sobre la opinión del deportista, ya que la admisión de cambios en la planificación de entrenamiento podría condicionar los objetivos de la misma. Sin embargo, el hecho de experimentar roles e ir tomando decisiones a lo largo del proceso de entrenamiento, hizo que el grado de implicación de los nadadores fuese muy elevado, lo que sin duda mejoró su experiencia de aprendizaje y entrenamiento. De este modo, se apreciaba que la inercia metodológica que ya reportaron Calderón, et al. (2013), también condicionó positivamente el incremento en la percepción de autonomía de los nadadores.

Además se encontró que la intervención provocó mejoras en el grado de disfrute y competencia percibida. En esta línea, también existe un amplio número de investigaciones realizadas en el contexto escolar que analizan esta variable (Hastie 
y Sinelnikov, 2006; MacPhail, et al., 2008; Mohr, Townsend, Bulger, et al., 2006; Wallhead y Ntoumanis, 2004). En ellas se muestran mejoras significativas del disfrute y la competencia percibida como consecuencia de las características de la Educación Deportiva. Si bien, es preciso tener en cuenta que el cambio de una metodología más directiva, en la que el nadador es mero reproductor, a otra en la que tiene un papel más activo, ya puede condicionar la mejora de estas variables independientemente del modelo que se utilice (Calderón, et al., 2013).

Otra de las variables de estudio que presentó mejoras, condicionadas en parte por el comportamiento de las dos variables anteriores, fue el grado de compromiso deportivo de los nadadores. Numerosos estudios sobre este modelo de enseńanza desarrollados en el contexto escolar ya han demostrado que el aumento en la percepción de autonomía por parte de los alumnos, contribuye a incrementar la adherencia, el compromiso y la implicación en las tareas (Hastie y Curtner-Smith, 2006; Kinchin, Wardle y Sprosen, 2004; MacPhaily Kinchin, 2004; Martínez de Ojeda, Calderón y Campos, 2012; Mowling, Brock y Hastie, 2006; Sinelnikov, et al., 2010).

En el presente estudio piloto, el modelo de ecuaciones estructurales confirma esta tendencia. Lo cual refuerza la tesis de que las características propias del modelo (roles, registro, evaluación, etc) y/o de otros programas de intervención de similares características, cuando se aplican en un contexto extraescolar o de club federado pueden fomentar un clima en los entrenamientos que estimule la adherencia y el compromiso en la práctica, tan importante hoy, dado el elevado declive de la participación federada en determinados deportes (Consejo Superior de Deportes, 2011).

Tal como se hipotetizó en el modelo de ecuaciones estructurales, el comportamiento autónomo predijo el grado de disfrute y competencia percibida, y a su vez el compromiso deportivo de los nadadores. Resultados coincidentes con los hallados en el contexto escolar por Méndez-Giménez, Fernández-Río, y Cecchini-Estrada (2013), que diseñaron un modelo de ecuaciones estructurales y verificaron la importancia de estas variables psicológicas para generar un clima motivacional que favorezca el aprendizaje. Resultados coincidentes con los hallados por Fernández-Río, Méndez-Giménez, y Cecchini-Estrada (2014), al analizar esta variable psicológica también en contexto escolar.

Además, es preciso destacar que tal como indican numerosos autores, tanto el clima motivacional trasmitido por el entrenador, como el modelo de enseńanza utilizado, se consideran predictores del estado psicológico de los deportistas, lo cual es importante en el contexto deportivo de iniciación y especialización ya que se estimulará un grado de disfrute adecuado entre los jóvenes deportistas (Almagro, Sáenz-López, González-Cutre y Moreno-Murcia, 2011; Balaguer, Gonzá- lez, Fabra, Castillo, Mercé, y Duda, 2012; Moreno, López de San Román, Martínez, Alonso, y González-Cutre, 2008; Quested y Duda, 2010; Torregrosa, Belando y Moreno-Murcia, 2013).

Se trata por tanto de seguir analizando las interrelaciones que se generan entre determinadas variables psicológicas para proporcionar un adecuado apoyo psicológico a los deportistas en formación (Rial, Marsillas, Isorna y Louro, 2013), que posibilite a medio y largo plazo un mejor rendimiento deportivo. Concretamente, en el contexto de natación federada de alto nivel, Fernández-Río, Cecchini, Méndez-Giménez, Fernández-García y Saavedra (2013), han corroborado que los entrenadores deben controlar determinadas variables de su intervención para conseguir climas de entrenamiento favorables que mantengan a los deportistas motivados de forma intrínseca durante las sesiones de entrenamiento.

En la misma línea que el estudio de Santi, Bruton, Pietrantoni, y Mellalieu (2014), que también aporta importancia al apoyo prestado entre los propios compañeros de entrenamiento. Los resultados obtenidos tras análisis del contenido, de las entrevistas y del diario, refuerzan todo lo anterior. Los nadadores percibían una mayor autonomía durante los entrenamientos, tal como reveló uno de ellos: "Ahora al entrenar con roles aprendemos a la misma vez que nadamos porque ponemos más de nuestra parte para que salga todo bien y consigamos mejorar, todos somos importantes".

Además durante el programa de intervención valoraron su papel activo en el grupo, lo que también pudo condicionar su grado de disfrute, y de competencia percibida. Una nadadora enfatizó lo siguiente: "Estas cuatro semanas para mi han sido muy buenas porque hemos entrenado demostrándonos que somos un gran equipo, nos hemos superado en cada entrenamiento y esto nos hace mejores nadadores". El propio entrenador aludió al comportamiento favorable de los nadadores en los entrenamientos. Tal como indicó en su diario: "Es increíble el cambio para ellos y para mí, porque ahora todos entrenan con mejor actitud, más positivos y con ganas de dar el máximo rendimiento en cada sesión". En resumen, los nadadores percibieron el programa de intervención como una forma innovadora de entrenar, que les permite tomar responsabilidades en su propio proceso de enseńanza-aprendizaje y rendimiento, lo cual les satisface y les hace sentir más competentes.

\section{Conclusiones}

Un programa de intervención diseñado teniendo en cuenta las características de la Educación Deportiva en el contexto federativo (en natación), se asocia a un comportamiento favorable de las variables percepción de autonomía y grado de disfrute y competencia percibida, tal como ocurre en el contexto escolar en la clase de educación física tras unidades didácticas que utilizan este modelo de enseñanza. También condiciona 
mejoras en el grado de compromiso deportivo de los nadadores que lo experimentaron. Por ello, las intervenciones metodológicas de este tipo, se presentan como una alternativa a los modelos de enseñanza tradicionales que frecuentemente se usan en el ámbito federativo y en deportes cíclicos como la natación. En futuras investigaciones se podrán analizar los efectos de la intervención a nivel longitudinal, con otros grupos de edad y teniendo en cuenta la interrelación de otras variables de tipo psicológico y/o de entrenamiento.

\section{Aplicaciones prácticas}

De entre las aplicaciones prácticas que se extraen del presente trabajo, y que podrán guiar intervenciones de este tipo, se consideran importantes las siguientes:

1) Es fundamental antes de comenzar con el programa de intervención se explique a los nadadores las características metodología a desarrollar. Deberán conocer muy bien las funciones de cada rol específico. Para ello el grado de conocimiento del modelo y la formación por parte del entrenador debe ser la adecuada.
2) Es importante que sea el entrenador quién cada semana asigne el rol a desarrollar por cada nadador. El criterio de selección que tendrá en cuenta podrá ser la personalidad de cada nadador, de forma que aquellos que puedan tender hacia la introversión, no se les asignen (al menos en las primeras semanas) roles con protagonismo (como el de entrenador).

3) Para recordar las características de las funciones de cada rol general y específico, se pondrán carteles en la piscina, en los que se detalle toda la información de forma gráfica.

4) Es fundamental que el entrenador se encuentre controlando el desarrollo de todas las sesiones de entrenamiento, para que se cumplan todas las variables que componen cada ejercicio (volumen, intensidad y tiempos de descanso), y con ello la planificación en general.

5) A lo largo del proceso de intervención, el entrenador estará más presente y ayudará a tomar determinadas decisiones a propósito de los roles y de los ejercicios a sus nadadores. Conforme avanzan las semanas, se debe percibir que la ayuda del entrenador se hace menos necesaria.

\section{Referencias}

1. Almagro, B. J., Sáenz-López, P., González-Cutre, D., y MorenoMurcia, J. A. (2011). Clima motivacional percibido, necesidades psicológicas y motivación intrínseca como predictores del compromiso deportivo en adolescentes. International Journal of Sport Science, 25(7) 250-265. doi:10.5232/ricyde2011.02501.

2. Anderson, J. C., y Gerbing, D. W. (1988). Structural equation modeling in practice: A review and recommended two-step approach. Psychological bulletin, 103(3), 411. doi: 10.1037/0033-2909.103.3.411.

3. Arias, J. L., Alonso, J. I., y Yuste, J. L. (2013). Propiedades psicométricas y resultados de la aplicación de la escala de disfrute y competencia percibida en baloncesto de iniciación. Universitas Psychologica, 12(3) 945-954. doi:10.11144/Javeriana.UPSY12-3.ppra.

4. Balaguer, I., González, L., Fabra, P., Castillo, I., Mercé, J., y Duda, J. L. (2012). Coache's interpersonal style, basic psychological needs and the well-and ill being of young soccer players: A longitudinal analysis. Journal of Sports Sciences, 30(15), 1619-1629. doi:10.1080/02640414.2 012.731517 .

5. Barbier, R. (1997). L'Approche Transversale. L'écoute sensible en sciencies humaines. París: Anthropos.

6. Bardin, L. (2002). Análisis de contenido. Madrid: Ediciones Akal.

7. Belando, N., Ferriz-Morell, R., y Moreno-Murcia, J. A. (2012). Validación de la escala de grado de compromiso deportivo en el contexto espanol. Motricidad. European Journal of Human Movement, 28, 111-124.

8. Bentler, P. M. (1989). EQS Structural Equations Program Manual. Los Angeles: BMDP Statistical Software.

9. Bompa, T. O. (2003). Periodización. Teoría y Metodología del entrenamiento. Barcelona: Editorial Hispano Europea.

10. Bunker, D., y Thorpe, R. (1982). A model for the teaching of games in secondary schools. Bulletin of Physical Education, 18, 5-8.

11. Calderón, A., Martínez de Ojeda, D., y Hastie, P. (2013). Valoración de alumnado y profesorado tras la experiencia práctica con dos metodologías de enseñanza en educación física. RICYDE. Revista Internacional de Ciencias del Deporte, IX(32), 137-153. doi: 10.5232/ ricyde2013.03204.
12. Calderón, A., Martínez de Ojeda, D., y Martínez, I. M. (2013). Influencia de la habilidad física percibida sobre la actitud del alumnado tras una unidad didáctica basada en Educación Deportiva. Retos. Nuevas Tendencias en Educación Física, Deporte y Recreación, 24,16-20.

13. Carretero-Dios, H., y Pérez, C. (2007). Normas para el desarrollo y revisión de estudios instrumentales: consideraciones sobre la selección de test en la investigación psicológica. International Journal of Clinical and Health Psychology, 7(3), 863-882.

14. Cervelló, E., Santos-Rosa, F. J., Jiménez, R., Nerea, A., y García, T. (2010). Motivación y ansiedad en jugadores de tenis. Motricidad. European Journal of Human Movement, 9.

15. Conde, C., Sáenz-López, Carmona, J., González-Cutre, D., Martínez, C., y Moreno, J. A. (2010). Validación del Cuestionario de Percepción de Soporte de la Autonomía en el Proceso de Entrenamiento (ASCQ) en jóvenes deportistas espańoles. Estudios de Psicología, 31(2), 145-157. doi: 10.1174/021093910804952250.

16. Consejo Superior de Deportes. (2011). Los hábitos deportivos de la población escolar en España. Underbau.

17. Dyson, B., y Casey, A. (2012). Cooperative Learning in Physical Education. A research-based approach. Routledge.

18. Ennis, C. D. (2014). The Role of Students and Content in Teacher Effectiveness. Research Quarterly for Exercise and Sport, 85(1), 6-13. do i.10.1080/02701367.2014.872979.

19. Ennis, C., y Chen, S. (2012). Interviews and focus groups. En Armour, K., Macdonald, D. (Ed.), Research Methods in Physical Education and Youth Sport. (p. 217-236). London: Routledge.

20. Fernández-Río, J., Cecchini, A., Méndez-Giménez, A., FernándezGarcía, B., y Saavedra, P. (2013). $2 \times 2$ Dominant achievement goal profiles in high-level swimmers. European Journal of Sport Science, $265-$ 272. doi:10.1080/17461391.2013.819383.

21. Fernández-Río, J., Méndez-Giménez, A., y Cecchini Estrada, J. A. (2014). A cluster analysis on student's perceived motivational climate. Implications on psycho-social variables. The Spanish Journal of Psychology, 17, E18. doi: 10.1017/sjp.2014.21. 
22. Gilbert, W., y Trudel, P. (2004). Analysis of coaching science published from 970-2001. Research Quarterly for Exercise and Sport, 75, 388399. doi:10.1080/02701367.2004.10609172.

23. Hastie, P. A., y Curtner-Smith, M. D. (2006). Influence of a hybrid Sport Education Teaching Games for Understanding unit on one teacher and his students. Physical Education \& Sport Pedagogy, 11(01), 1-27.

24. Hastie, P. A., Martínez de Ojeda, D., y Calderón, A. (2011). A review of research on Sport Education: 2004 to the present. Physical Education and Sport Pedagogy, 16(2), 103-132. doi:10.1080/17408989.2010.5 35202 .

25. Hastie, P. A., y Sinelkinov, O. A. (2006). Russian students participation in and perceptions of a season of Sport Education. European Physical Education Review, 12(2), 131-151. doi: 10.1177/1356336X06065166.

26. Hastie, P. A., Sinelnikov, O. A., y Guarino, A. J. (2009). The development of skill and tactical competencies during a season of badminton. European Journal of Sport Science, 9(3), 133-140. doi: $10.1080 / 17461390802542564$.

27. Jurado, M. D. (2011). El diario como instrumento de autoformación e investigación. Revista Qurriculum, 24, 173-200.

28. Kinchin, G. D., Macphail, A., y Ni Chroinin, D. (2009). Pupils' and teachers' perceptions of a culminating festival within a sport education season in irish primary schools. Physical Education \& Sport Pedagogy, 14(4), 391-406. doi: 10.1080/17408980802584982.

29. Kinchin, G. D., Wardle, S., y Sprosen, A. (2004). A survey of year 9 boys' perceptions of sport education in one English secondary school. Bulletin of Physical Education, 40(1), 27-40.

30. Lindsay, E. L. (2014). Effective Teaching in Physical Education: The View From a Variety of Trenches. Research Quarterly for Exercise and Sport, 85(1), 31-37. doi:10.1080/02701367.2014.873330.

31. Locke, L. (1977). Research on teaching physical education: New hope for a dismal science. Quest, 28, 2-16. doi: 10.1080/00336297.1977.10519895.

32. MacPhail, A., Gorely, T., Kirk, D., y Kinchin, G. (2013). Children's experiences of fun and enjoyment during a season of Sport Education. Research Quarterly for Exercise and Sport, 79(3), 344-355. doi: 10.1080/02701367.2008.10599498.

33. MacPhail, A., y Kinchin, G. (2004). The use of drawings as anevaluativetool: Students' experiences of sport education. Physical Education and Sport Pedagogy, 9(1), 87-108. doi: 10.1080/1740898042000208142.

34. Maglischo, E. W. (2009). Natación, técnica, entrenamiento y competición. Paidotribo.

35. Martínez de Ojeda, D., Calderón, A., y Campos, A. (2012). Percepción de aprendizaje y satisfacción en una unidad didáctica integrada mediante el modelo de educación deportiva. Cultura_Ciencia_Deporte, 21(7), 163-172. doi: 10.12800/ccd.v7i21.87.

36. Maxwell, J. A. (2013). Causal Explanation, Qualitative Research, and Scientific Inquiry in Education. Educational Researcher, 33(2), 3-11. doi: 10.3102/0013189X033002003.

37. Méndez-Giménez, A., Fernández-Río, J., y Cecchini-Estrada, J. A. (2013). Climas motivacionales, necesidades, motivación y resultados en Educación Física. Aula Abierta, 41(1), 63-72.

38. Metzler, M. W. (2014). Teacher Effectiveness Research in Physical Education: The Future Isn't What It Use to Be. Research Quarterly for Exercise and Sport, 85(1), 14-19. doi: 10.1080/02701367.2014.872932.

39. Mohr, D., Townsend, J., Bulger, S., Rairigh, R., y Mohr, C. (2006). Effect of a pedagogical approach to sport education season on sport literacy. Research Quarterly for Exercise and Sport, 77(Suppl.), A-67.

40. Moreno-Murcia, J. A., Cervelló-Gimeno, E., Marcos-Pardo, P. J., y Martín-Dantas, E. E. (2010). Importancia de la valoración del comportamiento autónomo del practicante para el disfrute en programas de ejercicio físico acuático. Cuadernos de Psicología del Deporte, 10(1), 57-70.
41. Moreno, J. A., López de San Román, M., Martínez, C., Alonso, N., y González-Cutre, D. (2008). Peers influence on exercise enjoyment: A self-determination theory approach. Journal of Sport Science and Medicine, 7, 23-31.

42. Mowling, C. M., Brock, S. J., y Hastie, P. A. (2006). Fourth grade students' drawing interpretations of a sport education soccer unit. Journal of Teaching in Physical Education, 25(1), 9-35.

43. Perugini, M., y Conner, M. T. (2000). Predicting and understanding behavioral volitions: The interplay between goals and behaviors. European Journal of Social Psychology, 30, 705-731. doi:10.1002/10990992(200009/10)30:5<705::AID-EJSP18>3.0.CO;2-\#.

44. Perlman, D. J. (2010). Change in affect and needs satisfaction for amotivated students within the sport education model. Journal of Teaching in Physical Education, 29(4), 433-445.

45. Perlman, D. J., y GocKarp, G. (2010). A self-determination perspective of the Sport Education model. Physical Education and Sport Pedagogy, 15(4), 401-418. doi: 10. 1080/17408980903535800.

46. Platonov, V. N., y Bulatova, M. M. (2007). La preparación física. Paidotribo.

47. Quested, E., y Duda, J. L. (2010). Exploring the social-environmental determinants of well-and ill-being in dancers: a test of basic needs theory. Journal of Sport \& Exercise Psychology, 32(1).

48. Rial, A., Marsillas, S., Isorna, M., y Louro, A. (2013). Recomendaciones para el apoyo psicológico a jóvenes deportistas en los centros de alto rendimiento. Revista Internacional de Ciencias del Deporte, IX(33) 252-268. doi: 10.5232/ricyde2013.03304.

49. Roberts, G. (2014). Time to Demonstrate How We Impact Student Learning. Research Quarterly for Exercise and Sport, 85(1), 27-30. doi: 10.1080/02701367.2013.872490.

50. Ruiz, M. A., Pardo, A., y San Martín, R. (2010). Modelos de ecuaciones estructurales. Papeles del Psicólogo, 31(1), 34-45.

51. Santi, G., Bruton, A., Pietrantoni, L., y Mellalieu, S. (2014). Sport commitment and participation in masters swimmers: The influence of coach and teammates. European Journal of Sport Science, 1-9. doi:10.10 80/17461391.2014.915990.

52. Schreiber, J. B., Nora, A., Stage, F. K., Barlow, E. A., y King, J. (2006). Reporting structural equation modeling and confirmatory factor analysis results: A review. The Journal of Educational Research, 99(6), 323-338. doi:10.3200/JOER.99.6.323-338.

53. Siedentop, D. (1994). Sport Education: Quality PE through positive sport experiences. Champaign, IL: Human Kinetics.

54. Silverman, S., y Manson, M. (2003). Research on teaching in physical education doctoral dissertations: a detailed investigation of focus, method, and analysis. Journal of Teaching in Physical Education, 22, 280-297.

55. Sinelnikov, O. A. (2009). Sport education for teachers: Professional development when introducing a novel curriculum model. European Physical Education Review, 15(1), 91-114. doi: 10.1177/1356336X09105213.

56. Sinelnikov. O. A., y Hastie, P. (2010). A motivational analysis of a season of Sport Education. Physical Education \& Sport Pedagogy, 15(1) 55-69. doi:10.1080/17408980902729362.

57. Sinelnikov, O. A., Hastie, P., y Prusak, K. A. (2007). Situational motivation in a season of sport education. ICHPER-SD Research Journal, 2(1), 43-47.

58. Solmon, M. A., y Garn, A. C. (2014). Effective teaching in physical education: using transportation metaphors to assess our status and drive our future. Research Quarterly for Exercise and Sport, 85(1), 20-26. doi: 10.1080/02701367.2013.872530.

59. Spittle, M., y Byrne, K. (2009). The influence of sport education on student motivation in physical education. Physical Education and Sport Pedagogy, 14(3), 253-266. doi: 10.1080/17408980801995239.

60. Thomas, J. R., Nelson, J. K., y Silverman, S. (2011). Research methods in physical activity (5th ed). Champaign, IL: Human Kinetics. 
61. Torregrosa, D., Belando, N., y Moreno-Murcia, J. A. (2013). Predicción de la satisfacción con la vida en practicantes de ejercicio físico saludable. Cuadernos de Psicología del Deporte, 14(1), 117-122. doi: $10.4321 /$ S1578-84232014000100014

62. Wallhead, T. L., Hagger, M., y Smith, D. T. (2010). Sport Education and extra-curricular sport participation: An examination using the trans-contextual model of motivation. Research Quarterly for Exercise and Sport, 82(4), 442-455. doi: 10.5641/027013610X13088600029256.

63. Wallhead, T.L., y Ntoumanis, N. (2004). Effects of a sport education intervention in student's motivational responses in physical education. Journal of Teaching in Physical Education, 23(1), 4-18.

64. Wallhead, T., y O'Sullivan, M. (2005). Sport education: Physical education for the new millennium?. Physical Education \& Sport Pedagogy, 10(2), 181-210. doi:10.1080/17408980500105098. 